\title{
The role of intestinal microbiota in the pathogenesis of NAFLD: starting points for intervention
}

Umberto Vespasiani-Gentilucci, Paolo Gallo, Antonio Picardi

Department of Medicine, Unit of Internal Medicine and Hepatology,

University Campus Bio-Medico, Rome, Italy

Submitted: 29 October 2015

Accepted: 5 February 2016

Arch Med Sci 2018; 14, 3: 701-706

DOI: https://doi.org/10.5114/aoms.2016.58831

Copyright $\odot 2016$ Termedia \& Banach

\section{Abstract}

In recent years, close links between intestinal microbiota and host metabolism have been recognized. Intestinal bacteria can participate in the extraction of calories from food, and circulation of bacterial products, in particular lipopolysaccharides (LPS), is responsible for the "metabolic endotoxemia", which contributes to insulin resistance and its complications, such as non-alcoholic fatty liver disease (NAFLD). Indeed, qualitative and quantitative intestinal dysbiotic changes have been clearly documented in NAFLD patients, and several mechanisms by which the intestinal microbiota can directly promote liver fat deposition, inflammation and fibrosis have also been described. Consistently, although with some differences concerning type and proportion of results, experimental and clinical studies are quite concordant in demonstrating beneficial effects of probiotic and/or prebiotic therapy in NAFLD. Although some physiopathological bases have been produced, major doubts still remain concerning how and when to intervene. Indeed, most of the available works were performed with mixtures of probiotics and/or prebiotics, and a baseline assessment of dysbiosis aimed at selecting the best candidates for treatment and predicting response has not been performed in any of the clinical studies in NAFLD. While future research is expected to solve these issues, the particularly favorable safety profile suggests that probiotic/prebiotic therapy could already be "tested" in NAFLD patients on an individual basis, at least once all the measures recommended by the latest guidelines have failed.

Key words: intestinal microbiota, non-alcoholic fatty liver disease, dysbiosis, small intestinal bacterial overgrowth, probiotics, prebiotics, lipopolysaccharides, lipopolysaccharide binding protein.

\section{Alterations of the intestinal microbiota during non-alcoholic fatty liver disease}

One important advance in the past years has been the recognition that there are close links between intestinal microbiota and host metabolism, and that the microbiota is a major environmental factor contributing to obesity and its complications, such as insulin resistance, type 2 diabetes, cardiovascular disease and non-alcoholic fatty liver disease (NAFLD) [1, 2]. Indeed, it has been demonstrated that intestinal bacteria can participate in the digestion of otherwise indigestible dietary polysaccharides, thereby influencing the amount of calories extracted from food [3]. Moreover, the consumption of a high fat diet is associated with loosening of intestinal tight junctions, increased intestinal permeability and elevated systemic levels of lipopolysaccharides (LPS) [4, 5]. This

\author{
Corresponding author: \\ Paolo Gallo \\ Department of Medicine \\ Unit of Internal \\ Medicine and Hepatology \\ University Campus \\ Bio-Medico \\ Via Alvaro del Portillo, 200 \\ 00128 Rome, Italy \\ Phone: +3906225411207 \\ E-mail: \\ paolo.gallo@unicampus.it
}


"metabolic endotoxemia" determines low-grade systemic inflammation, affecting insulin signaling and contributing to insulin-resistance and its complications [6].

Qualitative and quantitative intestinal dysbiotic changes have been clearly documented during NAFLD, both in patients with simple fatty liver and in those with non-alcoholic steatohepatitis (NASH) [7-14]. Microbiota samples from patients with NAFLD have a lower proportion of members of the Ruminococcaceae family than those from healthy subjects [7], and NASH patients show a significantly higher percentage of Clostridium coccoides than patients with simple steatosis [8]. Altogether, while some conflicting results have emerged between available studies addressing qualitative changes of intestinal microbiota in NAFLD patients, data concerning quantitative changes are quite concordant. Indeed, a number of works pointed to a high prevalence of small-intestinal bacterial overgrowth (SIBO) in patients with NAFLD [12-14].

There are several mechanisms by which the intestinal microbiota has been proven or suggested to contribute to liver fat deposition, inflammation and fibrosis. First, hepatotoxic bacterial products, i.e., pathogen-associated molecular patterns (PAMPs) and damage-associated molecular patterns (DAMPs), reaching the liver via the portal circulation, can activate specific toll-like receptors (TLRs) on different hepatic cells. In this context, the better recognized among bacterial constituents is LPS, which can activate TLR4 on Kupffer cells and hepatocytes, but also on cholangiocytes and on hepatic progenitor cells $[15,16]$, triggering the signaling cascade and the consequent secretion of several inflammatory cytokines. Recently, in patients with NAFLD, we demonstrated that TLR4 expression on bile duct/ductules and hepatic progenitor cells was significantly associated with inflammation, activation of fibrogenic cells and fibrosis, indicating the biliary clearance of excess LPS as a possible trigger of the inflammatory cascade in these cellular elements $[16,17]$. Consistently, LPS binding protein (LBP), a sensitive marker of LPS activity, was found to be elevated in NAFLD patients and to correlate with the stage of fibrosis [16]. Furthermore, LPS is not the only driver of systemic inflammation, and other bacterial products derived from gut microbiota can regulate insulin sensitivity and produce inflammation also by reducing anti-inflammatory strategies [18].

Second, the intestinal microflora of obese mice was found to be responsible for increased production of endogenous ethanol [19], which reaches the liver via portal blood, leads to hepatocyte triglyceride accumulation, and contributes to the production of reactive oxygen species and to in- flammation. In humans, the specific composition of the gut microbiome appears to be responsible for the abundance of alcohol-producing bacteria only among NASH patients, and not in healthy or obese non-NASH subjects [9].

Moreover, a high-fat diet was demonstrated to promote the formation of the intestinal microbiota, which converts dietary choline into methylamines, reducing plasma phosphatidylcholine and producing effects similar to those of the choline-deficient diet, a well-validated experimental model of NASH [20, 21].

Finally, more recently, also bile acids have been claimed as a possible contributor to the link between gut microbiota composition, dysmetabolism and NAFLD. Indeed, in murine models, it has been observed that bile acids not only play a key role in fat digestion and absorption, but also function as signaling molecules, binding to cellular receptors such as the bile-acid synthesis controlling nuclear receptor farnesoid $X$ receptor (FXR) and the G-protein coupled bile salt receptor TGR5 [22, 23]. Since FXR and TGR5 are involved in the modulation of glucose homeostasis [24, 25], the gut microbiota might regulate metabolism also by impacting the composition of the bile-acid pool [26]

Further evidence is required before the exact nature of the relationship between dysbiosis and NAFLD can be definitely solved. Indeed, it remains unclear whether only dysbiosis contributes to NAFLD or also NAFLD can favor dysbiosis, and whether dysbiosis is associated specifically with NAFLD or, more generally, with the metabolic disorders which subtend NAFLD development. The most consistent hypothesis is that an unbalanced (high-fat) diet both is directly responsible for liver fat accumulation and contributes to intestinal dysbiosis, which further promotes NAFLD and its progression. However, although further clarifications are certainly needed, a first message should pass: dysbiosis is associated with NAFLD and this relationship is harmful for the liver.

\section{Acting on intestinal microbiota may prove effective in NAFLD patients}

Experimental and clinical studies with probiotics and prebiotics support the role of intestinal microbiota in the pathogenesis of dysmetabolism and liver disease, and launch the modulation of dysbiosis as a possible therapeutic target in NAFLD [27].

Prebiotics are basically food for probiotics. Taking prebiotics helps probiotics work better and more efficiently. They are defined as a "non-digestible food ingredient that beneficially affects the host by selectively stimulating the growth and/or activity of one or a limited number of bacteria in the colon" [28]. Common prebiotics are 
inulin and carbohydrate fibers called oligosaccharides. On the other hand, probiotics are defined as preparations "containing viable, defined microorganisms in sufficient numbers, which alter the microflora (by implantation or colonization) in a compartment of the host exerting beneficial health effects" [29]. Finally, synbiotics are supplements that contain both probiotics and prebiotics. Probiotics are available commercially in many products but primarily as foods and dietary supplements. As with any health-related product, it is important for probiotics to be safe and effective. The agency charged with regulation of probiotic foods and food supplements, i.e., the European Food Safety Authority (EFSA), releases periodically the list of safe microbial cultures.

In the field of NAFLD, the most robust experience is that with VSL\#3, a mixture of probiotic bacteria including Lactobacilli, which was proven to attenuate liver inflammation, and sometimes also to reduce fibrosis, in different animal models [30-32]. Evidence also comes from human studies. In NAFLD and alcoholic cirrhosis patients, VSL\#3 improves plasma levels of lipid peroxidation markers [33], while Lactobacillus spp., Streptococcus thermophilus and Bifidobacterium bifidum decrease hepatic enzymes and liver fat content $[34,35]$. Furthermore, in a recently published randomized controlled trial, 4-month supplementation with VSL\#3 in obese children was found to significantly improve fatty liver severity, as determined by ultrasonography, and the effect was likely mediated by increased plasma levels of glucagon-like peptide 1 [36].

In mouse models, Lactobacilli, alone or mixed with other probiotics/prebiotics, were also shown to have direct metabolic effects, as demonstrated by their ability to increase hepatic peroxisome proliferator-activated receptor- $\alpha$ (PPAR- $\alpha$ ) activity [37], and to modulate insulin sensitivity [38]. Interestingly, a butyrate-producing probiotic (MIYAIRI 588) was recently found to reduce hepatic lipid deposition and inflammation, as well as serum endotoxin levels and insulin resistance, in a rat model of NAFLD [39]. Of note, patients with NAFLD have significantly increased gut permeability, as well as a higher prevalence of SIBO, compared to healthy subjects [12], and butyrate has been strongly implicated in the maintenance of intestinal barrier integrity [40].

After the 2007 Cochrane meta-analysis was not conclusive [41], mainly due to the limited number of available randomized controlled trials, other studies have been produced, and a more recent meta-analysis found probiotic treatment to reduce alanine aminotransferase (ALT) and improve insulin resistance in NAFLD patients [42]. A recent study in 32 NAFLD patients found a significant effect of probiotics on the reduction of ALT, cholesterol, triglyceride and body mass index (BMI) levels and, even more interestingly, their ability to potentiate weight loss induced by metformin [43]. However, the effect of probiotic/prebiotic treatment on body weight in humans certainly needs to be further explored, and these results should be considered preliminary.

Finally, also rifaximin, which is a virtually unabsorbable antibiotic with broad spectrum antimicrobial activity and an excellent safety profile, will likely carve itself a role in this field. It has already been shown that rifaximin significantly reduces plasma LPS levels and improves liver function tests in liver transplant candidates with alcoholic cirrhosis, and, currently, there is an ongoing randomized trial aimed at assessing the efficacy of rifaximin in NAFLD including the measurement of proinflammatory cytokine and endotoxin levels [44]. If this trial gives positive results, we will have proof of concept that intestinal decontamination, which has already been demonstrated to improve liver function and disease severity in patients with decompensated cirrhosis [45], could be a feasible and safe approach to prevent LPS-induced liver injury also in the context of NAFLD. In any case, before long-term efficacy and safety results with rifaximin, as well as with other unabsorbable antibiotics, are obtained, it seems imprudent to consider this approach in NAFLD patients, even on an individual basis.

Altogether, a significant amount of results from experimental studies, and accumulating evidence from human studies, imply that manipulation of intestinal microbiota by probiotics and/or prebiotics and/or rifaximin might exert beneficial effects in NAFLD and in the frequently associated metabolic disorders. Consistent with these data, the latest work demonstrated intestinal infusions of allogenic or autologous microbiota from lean donors to improve insulin sensitivity in subjects with metabolic syndrome [43].

\section{Starting points for intervention on intestinal microbiota in patients with NAFLD}

As pointed out above, qualitative and quantitative changes of the gut microbial community have been clearly documented in patients with NAFLD [7-14], and different possible mechanisms by which the intestinal microbiota can contribute to NAFLD pathogenesis have been demonstrated or suggested [9, 16, 19-21]. Although some physiopathological bases have been produced, major doubts still remain as to how and when to intervene. Indeed, most of the available studies were performed with a mixture of probiotics, frequently combined with one or more prebiotics, rendering the recognition of the specific agent responsible 
for the observed beneficial effects quite impossible. While waiting for further research aimed at discerning which effect should be attributed to each probiotic/prebiotic, we could already try to modulate intestinal microbiota using the mixtures of agents which have demonstrated beneficial effects in the better-designed available studies in NAFLD patients. However, even trying to simplify things by this approach, we would still face a significant unsolved question: is probiotic/prebiotic therapy effective in all NAFLD patients, or do we need a baseline screening test for prediction of the response? The truth is that we don't have an answer to this question, since a baseline assessment of dysbiosis has not been performed in any interventional study with this type of treatment in NAFLD patients. It is therefore desirable for future studies to be designed considering also a baseline assessment of dysbiosis, aiming to evaluate if and how the response to treatment can be predicted on an individual basis. However, how should we assess dysbiosis in an easy, reliable and cost-effective, i.e., clinically applicable, way? Pyrosequencing of $16 \mathrm{~S}$ ribosomal RNA, which has been essential for acquiring most of the knowledge concerning the gut bacterial composition in NAFLD, does not seem to be the most appropriate tool. In the same way, all the available methods to test for small intestinal bacterial overgrowth (SIBO), including direct aspiration and culture of the duodenal fluid and glucose/lactulose breath test, have substantial limitations, and no "gold standard" diagnostic test for the condition exists [46, 47]. When considering possible serum markers, it should be noted that various toxins produced by members of gut microbiota may enter the bloodstream via the enterohepatic circulation or the impaired gut barrier. Lipopolysaccharide is a major component of the Gram-negative bacterial wall, which can be detected and measured in the blood by the Limulus lysate assay. However, this test has a limited utility in the routine clinical setting, since LPS has a short half-life and high susceptibility to interfering substances [48]. LPS binding protein is an acute phase protein mainly produced by the liver in response to bacteremia or endotoxemia. It circulates in the blood, and its serum levels, which can be conveniently detected by commercial ELISA, indicate the amount of effective LPS load and the induced innate immune response [49]. Circulating LBP levels are increased in NAFLD patients $[16,50]$ and associated with the stage of fibrosis, as we recently demonstrated [16]. One limitation of LBP is that it only reflects Gram-negative bacteria and not Gram-positive translocation. However, its relatively long half-life, and the reliability and limited cost of the test, launch LBP as an attractive candidate to be further tested in the clinical ground.
In conclusion, to date, although some convincing knowledge on the implication of dysbiosis in the pathogenesis of NAFLD has been acquired, we still lack sufficient evidence to suggest when and how this knowledge should be applied. Future research is expected to further explore the physiopathological links between intestinal microbiota and NAFLD, to identify clinically relevant markers of dysbiosis and predictors of response, as well as to indicate the best agent, or combination of agents, to be used. Treatment of NAFLD should be first aimed at antagonizing insulin resistance, which is also central to all associated metabolic disturbances [51]: proper diet [52] and physical activity are the first keys to success. Currently, taking advantage of the optimal safety profile of probiotic/prebiotic therapy, we can only suggest that an approach with these agents in NAFLD could be explored in selected cases, after all the measures recommended by the latest guidelines [53], i.e., lifestyle interventions and eventually vitamin $E$ in non-diabetics or pioglitazone in diabetics, have failed.

\section{Conclusions}

Different pathogenic pathways are involved in the development of NAFLD, and, in recent years, growing evidence has indicated the participation of intestinal dysbiosis. While waiting for further research in order to understand when and how we should better modulate the gut intestinal microbiota in NAFLD patients, the very favorable risk profile already permits us to "test" probiotics/ prebiotics once the currently recommended therapeutic measures have failed.

\section{Conflict of interest}

The authors declare no conflict of interest.

\section{References}

1. Tremaroli V, Bäckhed F. Functional interactions between the gut microbiota and host metabolism. Nature 2012; 489: 242-9

2. Kau AL, Ahern PP, Griffin NW, Goodman AL, Gordon JI. Human nutrition, the gut microbiome and the immune system. Nature 2011; 474: 327-36.

3. Turnbaugh PJ, Ley RE, Mahowald MA, Magrini V, Mardis ER, Gordon J. An obesity-associated gut microbiome with increased capacity for energy harvest. Nature 2006; 444: 1027-131.

4. Cani PD, Bibiloni R, Knauf C, et al. Changes in gut microbiota control metabolic endotoxemia-induced inflammation in high-fat diet-induced obesity and diabetes in mice. Diabetes 2008; 57: 1470-81.

5. Cani PD, Possemiers S, Van de Wiele T, et al. Changes in gut microbiota control inflammation in obese mice through a mechanism involving GLP-2-driven improvement of gut permeability. Gut 2009; 58: 1091-103.

6. Tilg $H$, Moschen AR. Inflammatory mechanisms in the regulation of insulin resistance. Mol Med Camb Mass 2008; 14: 222-31. 
7. Raman M, Ahmed I, Gillevet PM, et al. Fecal microbiome and volatile organic compound metabolome in obese humans with nonalcoholic fatty liver disease. Clin Gastroenterol Hepatol 2013; 11: 868-75.

8. Mouzaki M, Comelli EM, Arendt BM, et al. Intestinal microbiota in patients with nonalcoholic fatty liver disease. Hepatol Baltim Md 2013; 58: 120-7.

9. Zhu L, Baker SS, Gill C, et al. Characterization of gut microbiomes in nonalcoholic steatohepatitis (NASH) patients: a connection between endogenous alcohol and NASH. Hepatology 2013; 57: 601-9.

10. Spencer MD, Hamp TJ, Reid RW, Fischer LM, Zeisel SH, Fodor AA. Association between composition of the human gastrointestinal microbiome and development of fatty liver with choline deficiency. Gastroenterology 2011; 140: 976-86.

11. Wong VW, Tse CH, Lam TT, et al. Molecular characterization of the fecal microbiota in patients with nonalcoholic steatohepatitis: a longitudinal study. PLoS One 2013; 8: e62885.

12. Miele L, Valenza V, La Torre G, et al. Increased intestinal permeability and tight junction alterations in nonalcoholic fatty liver disease. Hepatology 2009; 49: 1877-87.

13. Sabaté JM, Jouët P, Harnois F, et al. High prevalence of small intestinal bacterial overgrowth in patients with morbid obesity: a contributor to severe hepatic steatosis. Obes Surg 2008; 18: 371-7.

14. Wigg AJ, Roberts-Thomson IC, Dymock RB, McCarthy PJ, Grose RH, Cummins AG. The role of small intestinal bacterial overgrowth, intestinal permeability, endotoxaemia, and tumour necrosis factor alpha in the pathogenesis of non-alcoholic steatohepatitis. Gut 2001; 48: 206-11.

15. Vespasiani-Gentilucci U, Carotti S, Onetti-Muda A, et al. Toll-like receptor-4 expression by hepatic progenitor cells and biliary epithelial cells in HCV-related chronic liver disease. Mod Pathol 2012; 25: 576-89.

16. Vespasiani-Gentilucci U, Carotti S, Perrone G, et al. Hepatic toll-like receptor 4 expression is associated with portal inflammation and fibrosis in patients with NAFLD. Liver Int 2015; 35: 569-81.

17. Carotti S, Vespasiani-Gentilucci U, Perrone G, Picardi A, Morini S. Portal inflammation during NAFLD is frequent and associated with the early phases of putative hepatic progenitor cell activation. J Clin Pathol 2015; 68: 883-90.

18. Moschen AR, Kaser S, Tilg H. Non-alcoholic steatohepatitis: a microbiota-driven disease. Trends Endocrinol Metab 2013; 24: 537-45.

19. Cope K, Risby T, Diehl AM. Increased gastrointestinal ethanol production in obese mice: implications for fatty liver disease pathogenesis. Gastroenterology 2000; 119: 1340-7.

20. Dumas ME, Barton RH, Toye A, et al. Metabolic profiling reveals a contribution of gut microbiota to fatty liver phenotype in insulin-resistant mice. Proc Natl Acad Sci USA 2006; 103: 12511-6.

21. Abu-Shanab A, Quigley EM. The role of the gut microbiota in nonalcoholic fatty liver disease. Nat Rev Gastroenterol Hepatol 2010; 7: 691-701.

22. Hylemon PB, Zhou H, Pandak WM, Ren S, Gil G, Dent P. Bile acids as regulatory molecules. J Lipid Res 2009; 50: 1509-20.

23. Sinal CJ, Tohkin M, Miyata M, Ward JM, Lambert G, Gonzalez FJ. Targeted disruption of the nuclear receptor FXR/BAR impairs bile acid and lipid homeostasis. Cell 2000; 102: 731-44.
24. Prawitt J, Abdelkarim M, Stroeve JHM, et al. Farnesoid $X$ receptor deficiency improves glucose homeostasis in mouse models of obesity. Diabetes 2011; 60: 1861-71.

25. Thomas C, Gioiello A, Noriega L, et al. TGR5-mediated bile acid sensing controls glucose homeostasis. Cell Metab 2009; 10: 167-77.

26. Duboc H, Rajca S, Rainteau D, et al. Connecting dysbiosis, bile-acid dysmetabolism and gut inflammation in inflammatory bowel diseases. Gut 2013; 62: 531-9.

27. Paolella G, Mandato C, Pierri L, Poeta M, Di Stasi M, Vajro P. Gut-liver axis and probiotics: their role in non-alcoholic fatty liver disease. World J Gastroenterol 2014; 20: 15518-31.

28. Gibson GR, Roberfroid MB. Dietary modulation of the human colonic microbiota. Introducing the concept of prebiotics. J Nutr 1995; 125: 1401-12.

29. Havenaar R, Huis In't Veld MJH. Probiotics: a general view. In: Lactic Acid Bacteria in Health and Disease. Vol 1. Elsevier Applied Science Publishers, Amsterdam 1992.

30. Li Z, Yang S, Lin H, et al. Probiotics and antibodies to TNF inhibit inflammatory activity and improve nonalcoholic fatty liver disease. Hepatology 2003; 37: 343-50.

31. Ma X, Hua J, Li Z. Probiotics improve high fat diet-induced hepatic steatosis and insulin resistance by increasing hepatic NKT cells. J Hepatol 2008; 49: 821-30.

32. Velayudham A, Dolganiuc A, Ellis M, et al. VSL\#3 probiotic treatment attenuates fibrosis without changes in steatohepatitis in a diet-induced nonalcoholic steatohepatitis model in mice. Hepatology 2009; 49: 989-97.

33. Loguercio C, Federico A, Tuccillo C, et al. Beneficial effects of a probiotic VSL\#3 on parameters of liver dysfunction in chronic liver diseases. J Clin Gastroenterol 2005; 39: 540-3.

34. Aller R, De Luis DA, Izaola O, et al. Effect of a probiotic on liver aminotransferase in nonalcoholic fatty liver disease patients: a double blind randomized clinical trial. Eur Rev Med Pharmacol Sci 2011; 5: 1090-5.

35. Wong VW, Won GL, Chim AM, et al. Treatment of nonalcoholic steatohepatitis with probiotics. A proof-of-concept study. Ann Hepatol 2013; 12: 256-62.

36. Alisi A, Bedogni G, Baviera G, et al. Randomised clinical trial: the beneficial effects of VSL\#3 in obese children with non-alcoholic steatohepatitis. Aliment Pharmacol Ther 2014; 39: 1276-85.

37. Wagnerberger S, Spruss A, Kanuri G, et al. Lactobacillus casei Shirota protects from fructose-induced liver steatosis: a mouse model. J Nutr Biochem 2013; 24: 531-8.

38. Raso GM, Simeoli R, lacono A, et al. Effects of a Lactobacillus paracasei B21060 based synbiotic on steatosis, insulin signaling and toll-like receptor expression in rats fed a high-fat diet. J Nutr Biochem 2014; 25: 81-90.

39. Endo H, Niioka M, Kobayashi N, Tanaka M, Watanabe T. Butyrate-producing probiotics reduce nonalcoholic fatty liver disease progression in rats: new insight into the probiotics for the gut-liver axis. PloS One 2013; 8: e63388.

40. Suzuki T. Regulation of intestinal epithelial permeability by tight junctions. Cell Mol Life Sci 2013; 70: 631-59.

41. Lirussi F, Mastropasqua E, Orando S, Orlando R. Probiotics for non-alcoholic fatty liver disease and/or steatohepatitis. Cochrane Database Syst Rev 2007; 24: CD005165.

42. Ma YY, Li L, Yu CH, Shen Z, Chen LH, Li YM. Effects of probiotics on nonalcoholic fatty liver disease: a meta-analysis. World J Gastroenterol 2013; 19: 6911-8.

43. Shavakhi A, Minakari M, Firouzian H, Assali R, Hekmatdoost A, Ferns G. Effect of a probiotic and metformin on liver aminotransferases in non-alcoholic steatohep- 
atitis: a double blind randomized clinical trial. Int J Prev Med 2013; 4: 531-7.

44. NCT02009592. Efficacy of rifaximin on hepatosteatosis and steatohepatitis patients.

45. Kalambokis GN, Tsianos EV. Rifaximin reduces endotoxemia and improves liver function and disease severity in patients with decompensated cirrhosis. Hepatology 2012; 55: 655-6.

46. Vrieze A, Van Nood E, Holleman F, et al. Transfer of intestinal microbiota from lean donors increases insulin sensitivity in individuals with metabolic syndrome. Gastroenterology 2012; 143: 913-6.

47. Grace E, Shaw C, Whelan K, Andreyev HJN. Review article: small intestinal bacterial overgrowth: prevalence, clinical features, current and developing diagnostic tests, and treatment. Aliment Pharmacol Ther 2013; 38: 674-88.

48. Novitsky TJ. Limitations of the Limulus Amebocyte Lysate Test in demonstrating circulating lipopolysaccharides. Ann N Y Acad Sci 1998; 851: 416-21.

49. Schumann RR, Leong SR, Flaggs GW, et al. Structure and function of lipopolysaccharide binding protein. Science 1990; 249: 1429-31.

50. Ruiz AG, Casafont F, Crespo J, et al. Lipopolysaccharidebinding protein plasma levels and liver TNF-alpha gene expression in obese patients: evidence for the potential role of endotoxin in the pathogenesis of non-alcoholic steatohepatitis. Obes Surg 2007; 17: 1374-80.

51. Papaetis GS, Papakyriakou P, Panagiotou TN. Central obesity, type 2 diabetes and insulin: exploring a pathway full of thorns. Arch Med Sci 2015; 11: 463-82.

52. Bhagat U, Das UN. Potential role of dietary lipids in the prophylaxis of some clinical conditions. Arch Med Sci 2015; 11: 807-18.

53. Chalasani N, Younossi Z, Lavine JE, et al. The diagnosis and management of non-alcoholic fatty liver disease: practice Guideline by the American Association for the Study of Liver Diseases, American College of Gastroenterology, and the American Gastroenterological Association. Hepatology 2012; 55: 2005-23. 